\title{
Nash implementation via simple stochastic mechanisms: strategy space reduction
}

\author{
Michele Lombardi
}

Received: 12 October 2010 / Accepted: 19 March 2012 / Published online: 30 March 2012

(C) The Author(s) 2012. This article is published with open access at Springerlink.com

\begin{abstract}
Benoît and Ok (Games Econ Behav 64:51-67, 2008) show that in a society with at least three agents any weakly unanimous social choice correspondence (SCC) is Maskin's monotonic if and only if it is Nash-implementable via a simple stochastic mechanism (Benoit-Ok's Theorem). This paper fully identifies the class of weakly unanimous $S C C$ s that are Nash-implementable via a simple stochastic mechanism endowed with Saijo's message space specification (Saijo in Econometrica 56:693700,1988 ). It is shown that this class of SCC s is equivalent to the class of SCCs that are Nash-implementable via Benoît-Ok's Theorem.
\end{abstract}

Keywords Nash implementation - Strategy space reduction · Informational efficiency $\cdot$ Simple stochastic mechanisms

JEL Classification $\quad \mathrm{C} 72 \cdot \mathrm{D} 71$

\section{Introduction}

A central theorem in implementation theory when there are at least three agents is that Maskin monotonicity and the property of no-veto power are sufficient conditions for (exact) Nash implementability of social choice correspondences (SCCs) (Maskin 1999). Yet, unlikely Maskin monotonicity, a Nash-implementable SCC does

M. Lombardi ( $\square)$

Department of Quantitative Economics, Maastricht University, P. O. Box 616, 6200 MD Maastricht, The Netherlands

e-mail: m.lombardi@maastrichtuniversity.nl

M. Lombardi

Department of Economics, University of Surrey, Guildford, Surrey GU2 7XH, UK

e-mail: m.lombardi@surrey.ac.uk 
not necessarily meet the condition of no-veto power. ${ }^{1}$ Moreover, a number of interesting SCCs do not satisfy this condition. For example, individual rational, weak core, no-envy SCC s violate the condition of no-veto power. This has, in turn, motivated the study of the implications of modifying the canonical (Maskin-) mechanisms in various ways, and has turned in a number of alternatives to it.

By retaining the standard definition of Nash implementation, Benoît and Ok (2008) introduce mechanisms which allow for randomizations among social outcomes, labeled simple stochastic mechanisms. While these mechanisms allow for randomizations, these randomizations can take place only off the equilibrium path; in equilibrium, only pure social outcomes are chosen. As each agent must be able to evaluate uncertain prospects when lotteries are employed, Benoît and Ok (2008) extend each agent's preference relation defined over (pure) social outcomes in the following natural way: an agent prefers a social outcome $x$ to a randomization between that outcome and a dispreferred outcome $y$, and that randomization to the dispreferred outcome $y$. Under this preference extension and a domain restriction (top-coincidence condition), which is satisfied by typical environments arising in economic analysis, they show that any weakly unanimous $S C C$ is Nash-implementable if and only if it is Maskin monotonic; ${ }^{2}$ that is Benoit-Ok's Theorem. Therefore, they characterize Nash implementability without appealing to the auxiliary condition of no-veto power.

In this paper, we deal with the informational efficiency issue pertaining to BenoitOk's Theorem. These authors constructed game forms which are rather general. Not surprisingly, this generality comes to a price in terms of the informational demands imposed on agents in order to guarantee Nash implementability: Every agent is required to reveal not only his own preferences but also the preferences of all other agents, a social outcome and a positive integer not exceeding the number of agents (agent index). As the message space has efficiency implications for informational decentralization, we modify Benoît and Ok's mechanism in which these demands are significantly decreased.

Following Saijo (1988), we can image arranging agents in a clockwise circle facing inward, and require that each agent $\ell$ announces, a social outcome, an agent index, her own preferences and the preferences of the agent standing immediately to her left, that is, of agent $\ell+1$. Based on this idea, the paper shows that any weakly unanimous SCC is Maskin monotonic if and only if it is Nash-implementable via a simple stochastic mechanism endowed with Saijo's message space specification, under the mild domain restriction of top-coincidence condition.

From this result, it follows that there is an equivalence relationship between Nash implementation via simple stochastic mechanisms endowed with Saijo's message space specification and Benoît-Ok's Theorem. This result is in line with other results of Nash implementation theory. In particular, the reported equivalence relationship is analogous to the equivalence relationship between Nash implementation

\footnotetext{
1 An $S C C$ satisfies the condition of no-veto power if it selects all social outcomes which are top-ranked by all except at most one agent.

2 A social outcome is weakly unanimous if it is top-ranked by all agents and it is the unique top-ranked outcome for at least one agent. An SCC is weakly unanimous if it selects socially weakly unanimous outcomes.
} 
by $s$-mechanisms and Nash implementation by canonical mechanisms (Lombardi and Yoshihara 2010).

The paper is organized as follows. In Sect. 2, we introduce notation and definitions. In Sect. 3, Nash implementation via simple stochastic mechanisms is defined, a simple stochastic mechanism with the aforesaid strategy space reduction is constructed, and, finally, our Theorem is stated and proved. Section 4 concludes briefly.

\section{Preliminaries}

The environment is $\left(N, X, \mathcal{R}^{n}\right)$, where $N:=\{1, \ldots, n\}$ is a set of $n \geq 3$ agents, $X:=\{x, y, z, \ldots\}$ is the set of attainable alternatives (or outcomes), and $\mathcal{R}^{n}$ is the set of admissible preference profiles (or states of the world). Henceforth, we assume that the cardinality of $X$ is $\# X \geq 2$. Let $\mathcal{R}(X)$ be the set of all complete preorders on $X{ }^{3}$ Then $\mathcal{R}^{n}:=\mathcal{R}_{1} \times \cdots \times \mathcal{R}_{n}$ is a nonempty subset of the $n$-fold Cartesian product $\mathcal{R}^{n}(X):=\mathcal{R}_{1}(X) \times \cdots \times \mathcal{R}_{n}(X)$, where each $\mathcal{R}_{i}(X)$ is a copy of $\mathcal{R}(X)$. An element of $\mathcal{R}^{n}$ is denoted by $\succcurlyeq_{:}=\left(\succcurlyeq_{1}, \ldots, \succcurlyeq_{n}\right)$, where its $i$ th component is $\succcurlyeq_{i} \in \mathcal{R}_{i}(i \in N)$. The symmetric and asymmetric factors of any $\succcurlyeq_{i} \in \mathcal{R}_{i}$ are denoted $\sim_{i}$ and $\succ_{i}$, respectively. As usual, for any $x, y \in X$ and $\succcurlyeq_{i} \in \mathcal{R}_{i}(i \in N)$ we write $(x, y) \in \succcurlyeq_{i}$ as $x \succcurlyeq_{i} y$. For any preference profile $\succcurlyeq \in \mathcal{R}^{n}$ and any $i \in N$, let $(\succcurlyeq)_{-i}$ be the list of elements of $\succcurlyeq$ for all agents except $i$, i.e., $\left(\succcurlyeq_{-i}=\left(\succcurlyeq_{1}, \ldots, \succcurlyeq_{i-1}, \succcurlyeq_{i+1}, \ldots, \succcurlyeq_{n}\right)\right.$. Given a list $(\succcurlyeq)_{-i}$ and $\succcurlyeq_{i} \in \mathcal{R}_{i}$ of agent $i$, we denote by $\left(\left(\succcurlyeq_{-i}, \succcurlyeq_{i}\right)\right.$ the preference profile consisting of these $\succcurlyeq_{i}$ and $\left(\succcurlyeq_{-i}\right.$. For any $\left(\succcurlyeq_{i}, x\right) \in \mathcal{R}_{i} \times X$ let $L\left(\succcurlyeq_{i}, x\right)$ denote agent $i$ 's lower section of $\succcurlyeq_{i}$ at $x$, that is, $L\left(\succcurlyeq_{i}, x\right):=\left\{y \in X \mid x \succcurlyeq_{i} y\right\}$. For any $\succcurlyeq_{i} \in \mathcal{R}_{i}(i \in N)$, let $\max _{\succcurlyeq_{i}} X$ be the set of maximal alternatives according to $\succcurlyeq_{i}$, that is, $\max _{\succcurlyeq_{i}} X:=\left\{x \in X \mid x \succcurlyeq_{i}\right.$ yfor all $\left.y \in X\right\}$.

Fix an environment $\left(N, X, \mathcal{R}^{n}\right)$. A social choice correspondence (SCC) on $\mathcal{R}^{n}$ is a correspondence $F: \mathcal{R}^{n} \rightrightarrows X$ with $\emptyset \neq F(\succcurlyeq) \subseteq X$ for all $\succcurlyeq \in \mathcal{R}^{n}$. An SCC $F$ on $\mathcal{R}^{n}$ is monotonic if, for all $\succcurlyeq, \succcurlyeq^{\prime} \in \mathcal{R}^{n}$ with $x \in F(\succcurlyeq)$, we have that $x \in F\left(\succcurlyeq^{\prime}\right)$ whenever $L\left(\succcurlyeq_{i}, x\right) \subseteq L\left(\succcurlyeq_{i}^{\prime}, x\right)$ for all $i \in N$. $^{4}$ It satisfies no-veto power if, for all $\succcurlyeq \in \mathcal{R}$, we have that $x \in F(\succcurlyeq)$ whenever $x \in \max _{\succcurlyeq} X$ for at least $n-1$ agents. It is said weakly unanimous if, for all $\succcurlyeq \in \mathcal{R}^{n},\{x\}=\bigcap_{i \in N} \max _{\succcurlyeq_{i}} X$ implies $x \in F(\succcurlyeq)$.

A (canonical) mechanism is a pair $(M, h)$, where $M:=M_{1} \times \cdots \times M_{n}$, with each $M_{i}$ being a (nonempty) set, and $h: M \rightarrow X$. It consists of a message space $M$, where $M_{i}$ is the message space for agent $i \in N$, and an outcome function $h$. Let $m_{i} \in M_{i}$ denote a generic message (or strategy) for agent $i$. A message profile is denoted $m=\left(m_{1}, \ldots, m_{n}\right) \in M$. For any message profile $m \in M$ and $i \in N$, let $m_{-i}$ be the list $\left(m_{j}\right)_{j \in N \backslash\{i\}} \in \times_{j \in N \backslash\{i\}} M_{j}$ of elements of $m$ for all agents except $i$, i.e., $m_{-i}=\left(m_{1}, \ldots, m_{i-1}, m_{i+1}, \ldots, m_{n}\right)$. Denote the set of such $m_{-i}$ by $M_{-i}$ for each $i \in N$. Given a list $m_{-i} \in M_{-i}$ and a message $m_{i} \in M_{i}$ of agent $i$, we denote by $\left(m_{i}, m_{-i}\right)$ the message profile consisting of these $m_{i}$ and $m_{-i}$. Given the environment $\left(N, X, \mathcal{R}^{n}\right)$, a mechanism $(M, h)$ induces a class of (non-cooperative) normal form games $\left\{(M, h, R(\succcurlyeq)) \mid \succcurlyeq \in \mathcal{R}^{n}\right\}$, where $(M, h, R(\succcurlyeq))$ is a normal form game in which

\footnotetext{
3 A preorder is a reflexive and transitive binary relation.

4 Weak set inclusion is denoted by $\subseteq$.
} 
$N$ is the set of agent, $M_{i}$ is agent $i$ 's message space, and $R(\succcurlyeq)$ is a list $\left(R\left(\succcurlyeq_{i}\right)\right)_{i \in N}$ of complete preorders $R\left(\succcurlyeq_{i}\right)$, where each $R\left(\succcurlyeq_{i}\right)$ is defined on $M$ as $m R\left(\succcurlyeq_{i}\right) m^{\circ}$ if and only if $h(m) \succcurlyeq_{i} h\left(m^{\circ}\right)$. Given a normal form game $(M, h, R(\succcurlyeq))$, we say that $m \in M$ is a pure strategy Nash equilibrium (Nash equilibrium, hereafter) at the state $\succcurlyeq$ if and only if, for all $i \in N, m R\left(\succcurlyeq_{i}\right)\left(m_{i}^{\circ}, m_{-i}\right)$ for all $m_{i}^{\circ} \in M_{i}$. Let $\mathbf{N}(M, h, R(\succcurlyeq))$ denote the set of Nash equilibria of $(M, h, R(\succcurlyeq))$, whereas $\mathbf{N}_{h}(M, h, R(\succcurlyeq))$ represents its corresponding set of Nash equilibrium outcomes.

Given the environment $\left(N, X, \mathcal{R}^{n}\right)$, a mechanism $(M, h)$ is said to implement $F$ in Nash equilibria, or simply Nash-implements $F$, if and only if

$$
\mathbf{N}_{h}(M, h, R(\succcurlyeq))=F(\succcurlyeq) \quad \text { for all } \quad \succcurlyeq \in \mathcal{R}^{n} .
$$

If such a mechanism exists then $F$ is Nash-implementable.

In his seminal paper, given an environment $\left(N, X, \mathcal{R}^{n}\right)$ (in which at least three agents operate), Maskin (1999) proves that any SCC F is monotonic whenever it is Nash-implementable. Moreover, any SCC F which satisfies monotonicity and noveto power is Nash-implementable; this is Maskin's Theorem. Moore and Repullo (1990), Dutta and Sen (1991), Danilov (1992), Sjöström (1991) and Yamato (1992) refined Maskin's Theorem, providing necessary and sufficient conditions for Nash implementation.

\section{Nash implementation by simple stochastic mechanisms}

\subsection{Notations and definitions}

For any distinct $x, y \in X$, let $x \oplus y$ be an object which is not in $X$. Let $x:=x \oplus x$. Following Benoît and Ok (2008) $x \oplus y$ can be thought as a lottery that gives $x$ with probability $1 / 2$ and $y$ with probability $1 / 2$. Let $X^{*}:=\{x \oplus y \mid x, y \in X\}$. For any $i \in N$ and $\succcurlyeq_{i} \in \mathcal{R}_{i}(X)$, a preorder $\succcurlyeq_{i}^{*} \in \mathcal{R}_{i}\left(X^{*}\right)$ is said to be a monotonic extension of $\succcurlyeq_{i}$ if, for all $x, y \in X$,

i. $x \succcurlyeq_{i} y \Leftrightarrow x \succcurlyeq_{i}^{*} y$;

ii. $x \succ_{i} y \Rightarrow x \succ_{i}^{*} x \oplus y \succ_{i}^{*} y$ and $x \sim_{i} y \Rightarrow x \sim_{i}^{*} x \oplus y \sim_{i}^{*} y$.

Condition i. and part of condition ii. mean that $\succcurlyeq_{i}^{*}$ is an extension of $\succcurlyeq_{i}$ as they require that $\succcurlyeq_{i} \subseteq \succcurlyeq_{i}^{*}$ and $\succ_{i} \subseteq \succ_{i}^{*}$. Condition ii. also requires that an agent $i$ prefers an outcome $x$ to a randomization between $x$ and the dispreferred outcome $y$, and that randomization to the dispreferred outcome $y$.

Let $\mu\left(\succcurlyeq_{i}\right)$ be the set of all monotonic extensions of $\succcurlyeq_{i} \in \mathcal{R}_{i}$. Given a preference profile $\succcurlyeq \in \mathcal{R}^{n}$ define $\mu(\succcurlyeq):=\left\{\left(\succcurlyeq_{1}^{*}, \ldots, \succcurlyeq_{n}^{*}\right) \mid \succcurlyeq_{i}^{*} \in \mu\left(\succcurlyeq_{i}\right)\right.$ for $\left.i \in N\right\}$. Thus a generic element of $\mu(\succcurlyeq)$ is $\succcurlyeq^{*}:=\left(\succcurlyeq_{1}^{*}, \ldots, \succcurlyeq_{n}^{*}\right)$ which is a listing of monotonic extended preorders, one for each agent. Finally, for any $\mathcal{R}^{n} \subseteq \mathcal{R}^{n}(X)$, let $\mu\left(\mathcal{R}^{n}\right):=$ $\bigcup\left\{\mu(\succcurlyeq) \mid \succcurlyeq \in \mathcal{R}^{n}\right\}$.

A simple stochastic mechanism is a pair $(M, \eta)$, where $M:=M_{1} \times \cdots \times M_{n}$, with each $M_{i}$ being a (nonempty) set, and $\eta: M \rightarrow X^{*}$. It consists of a message space $M$, where $M_{i}$ is the message space for agent $i \in N$, and an outcome 
function $\eta$. Then, the mechanism $(M, \eta)$ assigns to each message profile $m \in M$ either an outcome in $X$ or a mixing of two outcomes in $X$. Given a simple stochastic mechanism $(M, \eta)$ and a message profile $m \in M$, for each agent $i \in N$ let $\eta\left(M_{i}, m_{-i}\right):=\left\{\eta\left(m_{i}^{\prime}, m_{-i}\right) \in X^{*} \mid\right.$ for some $\left.m_{i}^{\prime} \in M_{i}\right\}$ be the set outcomes attainable by this agent when all other agents are reporting $m_{-i}$.

Given the environment $\left(N, X, \mathcal{R}^{n}\right)$, a simple stochastic mechanism $(M, \eta)$ induces a class of (non-cooperative) normal form games $\left\{\left(M, \eta, R\left(\succcurlyeq^{*}\right)\right) \mid \succcurlyeq^{*} \in \mu\left(\mathcal{R}^{n}\right)\right\}$, where $\left(M, \eta, R\left(\succcurlyeq^{*}\right)\right)$ is a normal form game in which $N$ is the set of agents, $M_{i}$ is agent $i$ 's message space, and $R\left(\succcurlyeq^{*}\right)$ is a list $\left(R\left(\succcurlyeq_{i}^{*}\right)\right)_{i \in N}$ of preorders $R\left(\succcurlyeq_{i}^{*}\right)$, where each $R\left(\succcurlyeq_{i}^{*}\right)$ is defined on $M$ as $m R\left(\succcurlyeq_{i}^{*}\right) m^{\circ}$ if and only if $\eta(m) \succcurlyeq_{i}^{*} \eta\left(m^{\circ}\right)$. Given a normal form game $\left(M, \eta, R\left(\succcurlyeq^{*}\right)\right)$, the set of Nash equilibrium strategy profiles is denoted by $\mathbf{N}\left(M, \eta, R\left(\succcurlyeq^{*}\right)\right)$, whereas $\mathbf{N}_{\eta}\left(M, \eta, R\left(\succcurlyeq^{*}\right)\right)$ represents the corresponding set of Nash equilibrium outcomes. We omit formal definitions.

Given the environment $\left(N, X, \mathcal{R}^{n}\right)$, a simple stochastic mechanism $(M, \eta)$ is said to implement $F$ in Nash equilibria, or simply Nash-implements $F$, if and only if

$$
\mathbf{N}_{\eta}\left(M, \eta, R\left(\succcurlyeq^{*}\right)\right)=F(\succcurlyeq) \quad \text { for all } \succcurlyeq \in \mathcal{R}^{n} \text { and } \succcurlyeq^{*} \in \mu(\succcurlyeq)
$$

If such a simple stochastic mechanism exists then $F$ is Nash-implementable by a simple stochastic mechanism.

We make the following very weak regularity assumptions on environments $\left(N, X, \mathcal{R}^{n}\right)$. An environment $\left(N, X, \mathcal{R}^{n}\right)$ is said to satisfy the top-coincidence condition if, for any $\succcurlyeq \in \mathcal{R}^{n}$ and any $I \subseteq N$, with \#I=n-1, the set $\bigcap_{i \in I} \max _{\succcurlyeq_{i}} X$ is at most a unit set (Benoît and Ok 2008). Next, we state Benoît-Ok's Theorem.

Theorem Theorem (Benoît and Ok 2008). Let $\left(N, X, \mathcal{R}^{n}\right)$ be an environment satisfying the top-coincidence condition. Then, any weakly unanimous SCC F on $\mathcal{R}^{n}$ is monotonic if and only if it is Nash-implementable by a simple stochastic mechanism with message space $M_{i}=\mathcal{R}^{n} \times X \times N$ for each agent $i \in N$.

\subsection{Theorem}

Fix the environment $\left(N, X, \mathcal{R}^{n}\right)$ and let $(M, \eta)$ be a simple stochastic mechanism, where the message space of agent $i(\in N)$ is $M_{i}=\mathcal{R}_{i} \times \mathcal{R}_{i+1} \times X \times N$. Fix any $m \in M, \unlhd \in \mathcal{R}^{n}$, and $x \in X$, and let $m_{i}=\left(\unlhd_{i}^{i}, \unlhd_{i+1}^{i}, x^{i}, k^{i}\right) \in M_{i}$, where $i+1=1$ if $i=n$, and where the announcement of agent $i \in N$ about agent $j$ 's preferences is $\unlhd_{j}^{i}$. We say that the message profile $m \in M$ is:

i. consistent with $\unlhd$ and $x$ if, for all $j \in N, \unlhd_{j}^{j}=\unlhd_{j}^{j-1}=\unlhd_{j}$ and $x^{j}=x$, where $j-1=n$ if $j=1$.

ii. $m_{-i}$ consistent with $x$ and $\unlhd$, where $i \in N$, if for all $j \in N: x^{j}=x$, and for all $j \in N \backslash\{i, i+1\}: \unlhd_{j}^{j}=\unlhd_{j}^{j-1}=\unlhd_{j}, \unlhd_{i}^{i-1}=\unlhd_{i}, \unlhd_{i+1}^{i+1}=\unlhd_{i+1}$, and $\left[\unlhd_{i}^{i} \neq \unlhd_{i}\right.$ or $\left.\unlhd_{i+1}^{i} \neq \unlhd_{i+1}\right]$, where $\ell-1=n$ if $\ell=1$ for $\ell \in\{i, j\}$. 
iii. $m_{-i}$ quasi-consistent with $x$ and $\unlhd$, where $i \in N$, if for all $j \in N \backslash\{i\}: x^{j}=$ $x \neq x^{i}$, and for all $j \in N \backslash\{i, i+1\}: \unlhd_{j}^{j}=\unlhd_{j}^{j-1}=\unlhd_{j}, \unlhd_{i}^{i-1}=\unlhd_{i}$ and $\unlhd_{i+1}^{i+1}=\unlhd_{i+1}$, where $\ell-1=n$ if $\ell=1$ for $\ell \in\{i, j\}$.

The idea of our simple stochastic mechanism is similar in structure to the one used in Saijo (1988). Let us define the outcome function $\eta: M \rightarrow X^{*}$ as follows: For any $m \in M$,

Rule 1: $m$ is consistent with $x$ and $\unlhd \in \mathcal{R}^{n}$, where $x \in F(\unlhd)$, then $\eta(m)=x$.

Rule 2: For some $i \in N, m$ is $m_{-i}$ consistent with $x$ and $\unlhd \in \mathcal{R}^{n}$, where $x \in F(\unlhd)$, then $\eta(m)=x$

Rule 3: For some $i \in N, m$ is $m_{-i}$ quasi-consistent with $x$ and $\unlhd \in \mathcal{R}^{n}$, where $x \in F(\unlhd)$, and $L\left(\unlhd_{i}^{i-1}, x\right) \neq X$, then

$$
\eta(m)= \begin{cases}x^{i} \oplus x & \text { if } x^{i} \in L\left(\unlhd_{i}^{i-1}, x\right) \\ x & \text { otherwise. }\end{cases}
$$

Rule 4: Otherwise, $\eta(m)=x^{\ell(m)}$ where $\ell(m):=\sum_{j \in N} k^{j}(\bmod n) .^{5}$

The following properties are implied by the above rules:

1. If all agents make the same outcome announcement, then the unanimously announced outcome is the outcome of the mechanism;

2. In Rules $2-3$, agent $i$ is a deviator. In Rule 2, agent $i$ is not necessarily the only deviator whenever there is exactly one break in the preference announcement profile between agent $i$ 's preference announcement and that of agent $i-1$, i.e., $\unlhd_{i}^{i} \neq \unlhd_{i}^{i-1}=\unlhd_{i}$ and $\unlhd_{i+1}^{i}=\unlhd_{i+1}^{i+1}=\unlhd_{i+1}$. Indeed, agents $i-1$ and $i$ could be deviators if

$$
x \in F\left(\unlhd_{1}^{1}, \ldots, \unlhd_{i-1}^{i-1}, \unlhd_{i}^{i-1}, \unlhd_{i+1}^{i+1}, \ldots, \unlhd_{n}^{n}\right)
$$

and

$$
x \in F\left(\unlhd_{1}^{1}, \ldots, \unlhd_{i-2}^{i-2}, \unlhd_{i-1}^{i-2}, \unlhd_{i}^{i}, \ldots, \unlhd_{n}^{n}\right) .
$$

In Rule 3, however, as agent $i$ is the only agent reporting an outcome different from that reported by all others the mechanism identifies him as the unique deviator;

3. In Rule 3, deviator $i$ 's preference announcement $\left(\unlhd_{i}^{i}, \unlhd_{i+1}^{i}\right)$ does not affect the evaluation of the $S C C F$ as it does not enter into the evaluation of the preference announcement profile

$$
\left(\unlhd_{1}^{1}, \ldots, \unlhd_{i-1}^{i-1}, \unlhd_{i}^{i-1}, \unlhd_{i+1}^{i+1}, \ldots, \unlhd_{n}^{n}\right)=\left(\unlhd_{1}, \ldots, \unlhd_{i-1}, \unlhd_{i}, \unlhd_{i+1}, \ldots, \unlhd_{n}\right) ;
$$

\footnotetext{
5 It is clear that if the remainder is zero the winner of the game is agent $n$.
} 
4. If Rule 3 applies to $m$, then the outcome is a lottery $x^{i} \oplus x$ whenever deviator $i$ 's outcome is no better than the outcome $x$ announced by others under the preference for $i$ announced by the agent $i-1$, i.e., $\unlhd_{i}^{i-1}$;

5. In Rule 4, agent $i$ can appropriately choose an integer index to designate himself or some other agent as the winner of the modulo game given other agents' integer announcements.

The mechanism employs the idea due to Saijo (1988) to cover twice each agent's preferences: agent $i$ 's preferences are covered by his own announcement and by that of agent $i-1$. This allows us to find a preference profile which is unaffected by the deviator's preference announcement. In Rules 2-3, even though the deviator sends a different message announcement he can never induce Rule 4 . The outcome is confined to a lottery between the outcome $x$ announced by others and that announced by the deviator whenever the latter is in $L\left(\unlhd_{i}^{i-1}, x\right)$. As usual the deviator's lower contour set is evaluated not by his own preference announcement $\unlhd_{i}^{i}$, but by that of his neighbor $\unlhd_{i}^{i-1}$.

We are now ready to verify that the above simple stochastic mechanism Nash-implements every weakly unanimous $S C C F$ whenever the implementation environment satisfies the condition of top-coincidence.

Theorem Let $\left(N, X, \mathcal{R}^{n}\right)$ be an environment satisfying the top-coincidence condition. Let $F$ be a weakly unanimous SCC. Then, $F$ is monotonic if and only if a simple stochastic mechanism $(M, \eta)$ defined by Rules $1-4$ with message space $M_{i}=$ $\mathcal{R}_{i} \times \mathcal{R}_{i+1} \times X \times N$ implements $F$ in Nash equilibria.

\subsection{Proof of Theorem}

Let $\left(N, X, \mathcal{R}^{n}\right)$ be an environment satisfying the top-coincidence condition. The proof of the "If" part of Theorem is checked in Benoît and Ok (2008). Let $F$ on $\mathcal{R}^{n}$ be a weakly unanimous SCC satisfying monotonicity. Let $\diamond \in N$ be an arbitrary integer. We show that for any $\succcurlyeq \in \mathcal{R}^{n}$ and any $\succcurlyeq^{*} \in \mu(\succcurlyeq): F(\succcurlyeq)=\mathbf{N}_{\eta}(M, \eta, R(\succcurlyeq *))$.

Lemma 1 Let $\succcurlyeq \in \mathcal{R}^{n}$ and $\succcurlyeq^{*} \in \mu(\succcurlyeq)$. Then, $F(\succcurlyeq) \subseteq \mathbf{N}_{\eta}\left(M, \eta, R\left(\succcurlyeq^{*}\right)\right)$.

Proof of Lemma 1 Let $x \in F(\succcurlyeq)$. Let $m_{i}=\left(\succcurlyeq_{i}^{i}, \succcurlyeq_{i+1}^{i}, x^{i}, \diamond\right)=\left(\succcurlyeq_{i}, \succcurlyeq_{i+1}, x, \diamond\right)$ for each $i \in N$. By Rule 1 it follows that $\eta(m)=x$. Moreover, for each $i \in N$ as $L\left(\succcurlyeq_{i}^{i-1}, x\right)=L\left(\succcurlyeq_{i}, x\right)$ and $\succcurlyeq_{i}^{*}$ is a monotonic extension of $\succcurlyeq_{i}$ it follows that $x \succcurlyeq_{i}^{*} x^{i} \oplus x$ for any $x^{i} \in L\left(\succcurlyeq_{i}, x\right)$. Therefore, $\eta\left(M_{i}, m_{-i}\right) \subseteq L\left(\succcurlyeq_{i}^{*}, x\right)$ and so $x \in \mathbf{N}_{\eta}\left(M, \eta, R\left(\succcurlyeq^{*}\right)\right)$, as sought.

Lemma 2 Let $\left(N, X, \mathcal{R}^{n}\right)$ be an environment satisfying the top-coincidence condition. Let $F$ on $\mathcal{R}^{n}$ be a weakly unanimous and monotonic SCC. Then, for any $\succcurlyeq \in \mathcal{R}$ and any $\succcurlyeq^{*} \in \mu(\succcurlyeq): \mathbf{N}_{\eta}\left(M, \eta, R\left(\succcurlyeq^{*}\right)\right) \subseteq F(\succcurlyeq)$.

Proof of Lemma 2 Let $m$ be a Nash equilibrium message profile of $\mathbf{N}\left(M, \eta, R\left(\succcurlyeq^{*}\right)\right)$. We show that $\eta(m) \in F(\succcurlyeq)$. Consider the following cases. 
Suppose that $m$ falls into Rule 1 . Then, $m$ is consistent with $x$ and $\unlhd \in \mathcal{R}^{n}$, where $x \in F(\unlhd)$. Thus, $\eta(m)=x$. Take any agent $i \in N$. Suppose that $L\left(\unlhd_{i}^{i-1}, x\right) \neq X$. For any $y \in L\left(\unlhd_{i}^{i-1}, x\right) \backslash\{x\}$, changing his message announcement $m_{i}$ into $m_{i}^{\circ}=$ $\left(\unlhd_{i}^{\circ i}, \unlhd_{i+1}^{\circ i}, y, \diamond\right) \in M_{i}$ agent $i$ can alter the current outcome to a lottery $x \oplus y=$ $\eta\left(m_{i}^{\circ}, m_{-i}\right)$ by Rule 3. However, $x \succcurlyeq_{i}^{*} x \oplus y$ as $m \in \mathbf{N}\left(M, \eta, R\left(\succcurlyeq^{*}\right)\right)$. Since $\succcurlyeq_{i}^{*}$ is a monotonic extension of $\succcurlyeq_{i}$, we have that $x \succcurlyeq_{i} y$. Otherwise, let us consider the case that $L\left(\unlhd_{i}^{i-1}, x\right)=X$. Thus, for any $y \in L\left(\unlhd_{i}^{i-1}, x\right) \backslash\{x\}$, by changing the announcement $m_{i}$ into $m_{i}^{\circ}=\left(\unlhd_{i}^{\circ i}, \unlhd_{i+1}^{\circ i}, y, k^{\circ i}\right) \in M_{i}$ agent $i$ can trigger the modulo game. To attain the outcome $y$ agent $i$ has only to adjust the integer index so that he becomes the winner of the modulo game. Since $m \in \mathbf{N}\left(M, \eta, R\left(\succcurlyeq^{*}\right)\right)$ and $\succcurlyeq_{i}^{*}$ is a monotonic extension of $\succcurlyeq_{i}$, it follows $x \succcurlyeq_{i} y$. Because it holds for any $y \in$ $L\left(\unlhd_{i}, x\right) \backslash\{x\}$ and any $i \in N$, it follows that $L\left(\unlhd_{i}^{i-1}, x\right)=L\left(\unlhd_{i}, x\right) \subseteq L\left(\succcurlyeq_{i}, x\right)$ for all $i \in N$. Monotonicity implies that $x \in F(\succcurlyeq)$.

Suppose that $m$ falls into Rule 2. Then $m$ is $m_{-i}$ consistent with $x$ and $\unlhd \in \mathcal{R}^{n}$, where $x \in F(\unlhd)$. Thus, $\eta(m)=x$. We proceed according to the following sub-cases: (1) $\unlhd_{i}^{i} \neq \unlhd_{i}$ and $\unlhd_{i+1}^{i} \neq \unlhd_{i+1}$, (2) $\unlhd_{i}^{i} \neq \unlhd_{i}$ and $\unlhd_{i+1}^{i}=\unlhd_{i+1}$ and (3) $\unlhd_{i}^{i}=\unlhd_{i}$ and $\unlhd_{i+1}^{i} \neq \unlhd_{i+1}$.

Sub-case 2.1. $\unlhd_{i}^{i} \neq \unlhd_{i}$ and $\unlhd_{i+1}^{i} \neq \unlhd_{i+1}$

Then agent $i \in N$ is the unique deviator. Any other agent $j \in N \backslash\{i\}$ can attain any $y \in X \backslash\{x\}$ by inducing Rule 4 , so that $x \in \max _{\succcurlyeq_{j}} X$ by our supposition that $m \in \mathbf{N}\left(M, \eta, R\left(\succcurlyeq^{*}\right)\right)$. Let us consider agent $i$. Suppose that $L\left(\unlhd_{i}^{i-1}, x\right) \neq X$. Take any $y \in L\left(\unlhd_{i}^{i-1}, x\right) \backslash\{x\}$. By changing his message announcement $m_{i}$ into $m_{i}^{\circ}=\left(\unlhd_{i}^{\circ i}, \unlhd_{i+1}^{\circ i}, y, \diamond\right) \in M_{i}$ agent $i$ can alter the current outcome to a lottery $x \oplus y=\eta\left(m_{i}^{\circ}, m_{-i}\right)$ by Rule 3. However, $x \succcurlyeq_{i}^{*} x \oplus y$ as $m \in \mathbf{N}\left(M, \eta, R\left(\succcurlyeq^{*}\right)\right)$. It follows from the definition of $\succcurlyeq_{i}^{*}$ that $x \succcurlyeq_{i} y$. Otherwise, let $L\left(\unlhd_{i}^{i-1}, x\right)=X$. Thus, for any $y \in L\left(\unlhd_{i}^{i-1}, x\right) \backslash\{x\}$, by changing the announcement $m_{i}$ into $m_{i}^{\circ}=$ $\left(\unlhd_{i}^{\circ i}, \unlhd_{i+1}^{\circ i}, y, k^{\circ i}\right) \in M_{i}$ agent $i$ can trigger the modulo game. To attain the outcome $y$ agent $i$ has only to adjust the integer index $k^{\circ i}$ so that $\eta\left(m_{-i}, m_{i}^{\circ}\right)=y$. Since $m \in \mathbf{N}\left(M, \eta, R\left(\succcurlyeq^{*}\right)\right)$ and $\succcurlyeq_{i}^{*}$ is a monotonic extension of $\succcurlyeq_{i}$, it follows $x \succcurlyeq_{i} y$. Since it holds for any $y \in L\left(\unlhd_{i}, x\right) \backslash\{x\}$, it follows that $L\left(\unlhd_{i}^{i-1}, x\right)=L\left(\unlhd_{i}, x\right) \subseteq$ $L\left(\succcurlyeq_{i}, x\right)$. Again, monotonicity implies that $x \in F(\succcurlyeq)$.

Sub-case 2.2. $\unlhd_{i}^{i} \neq \unlhd_{i}$ and $\unlhd_{i+1}^{i}=\unlhd_{i+1}$

We distinguish whether $x \in F\left(\unlhd^{\prime}\right)$, where $\unlhd^{\prime}:=\left((\unlhd)_{-i}, \unlhd_{i}^{i}\right)$, or not. Suppose that $x \notin F\left(\unlhd^{\prime}\right)$. Then agent $i$ is the unique deviator. The same reasoning used above 
for sub-case 2.1 carries over into this sub-case, so that $x \in F(\succcurlyeq)$. Otherwise, let $x \in F\left(\unlhd^{\prime}\right)$. Agent $j \in N \backslash\{i-1, i\}$ can attain any other $y \in X \backslash\{x\}$ by inducing Rule 4, so that $x \in \max _{\succcurlyeq_{j}} X$ by our supposition that $m \in \mathbf{N}\left(M, \eta, R\left(\succcurlyeq^{*}\right)\right)$. Consider agent $i-1$. Suppose that $L\left(\unlhd_{i-1}^{i-2}, x\right) \neq X$. For any $y \in L\left(\unlhd_{i-1}^{i-2}, x\right) \backslash\{x\}$, changing his message announcement $m_{i-1}$ into $m_{i-1}^{\circ}=\left(\unlhd_{i-1}^{\circ i-1}, \unlhd_{i}^{\circ i-1}, y, \diamond\right) \in M_{i-1}$ agent $i-1$ can alter the current outcome to a lottery $x \oplus y=\eta\left(m_{i-1}^{\circ}, m_{-(i-1)}\right)$ by Rules 3. Since $m \in \mathbf{N}\left(M, \eta, R\left(\succcurlyeq^{*}\right)\right)$ we have that $x \succcurlyeq_{i-1}^{*} x \oplus y$, and so $x \succcurlyeq_{i-1} \quad y$ as $\succcurlyeq_{i-1}^{*}$ is a monotonic extension of $\succcurlyeq_{i-1}$. Otherwise, let $L\left(\unlhd_{i-1}^{i-2}, x\right)=X$. Thus, for any $y \in L\left(\unlhd_{i-1}^{i-2}, x\right) \backslash\{x\}$, by changing the announcement $m_{i-1}$ into $m_{i-1}^{\circ}=\left(\unlhd_{i-1}^{\circ i-1}, \unlhd_{i}^{\circ i-1}, y, k^{\circ i-1}\right) \in M_{i-1}$ agent $i-1$ can trigger the modulo game. To attain the outcome $y$ agent $i-1$ has only to adjust the integer index $k^{\circ i-1}$ so that $\eta\left(m_{-(i-1)}, m_{i-1}^{\circ}\right)=y$. Since $m \in \mathbf{N}\left(M, \eta, R\left(\succcurlyeq^{*}\right)\right)$ and $\succcurlyeq_{i-1}^{*}$ is a monotonic extension of $\succcurlyeq_{i-1}$, it follows $x \succcurlyeq_{i-1} y$. Since it holds for any $y \in L\left(\unlhd_{i-1}^{i-2}, x\right)=L\left(\unlhd_{i-1}^{i-1}, x\right)$, it follows that $L\left(\unlhd_{i-1}, x\right) \subseteq L\left(\succcurlyeq_{i-1}, x\right)$. By applying the same reasoning for agent $i$ where $y \in L\left(\unlhd_{i}^{i-1}, x\right)=L\left(\unlhd_{i}, x\right)$ we have that $L\left(\unlhd_{i}, x\right) \subseteq L\left(\succcurlyeq_{i}, x\right)$. Maskin monotonicity implies that $x \in F(\succcurlyeq)$.

Sub-case 2.3. $\unlhd_{i}^{i}=\unlhd_{i}$ and $\unlhd_{i+1}^{i} \neq \unlhd_{i+1}$

We distinguish whether $x \in F\left(\unlhd^{\prime}\right)$, where $\unlhd^{\prime}:=\left((\unlhd)_{-(i+1)}, \unlhd_{i+1}^{i}\right)$, or not. Again, if $x \notin F\left(\unlhd^{\prime}\right)$ then agent $i$ is the unique deviator, and by the same reasoning used in sub-case 2.1 we have that $x \in F(\succcurlyeq)$. Otherwise, let $x \in F\left(\unlhd^{\prime}\right)$. Then $m$ is $m_{-(i+1)}$ consistent with $x$ and $\unlhd^{\prime}$. It follows from the sub-case 2.2 that $x \in F(\succcurlyeq)$.

Suppose that $m$ falls into Rule 3. Then $m$ is $m_{-i}$ quasi-consistent with $x$ and $\unlhd \in R^{n}$, where $x \in F(\unlhd)$, and $L\left(\unlhd_{i}^{i-1}, x\right) \neq X$. We proceed according to the following steps: (1) $\eta(m)=x$, (2) $x \in \max _{\succcurlyeq j} X$ for all $j \in N \backslash\{i\}$, and (3) $x \in F(\succcurlyeq)$.

Step 1. $\eta(m)=x$

It is obvious that $\eta(m)=x$ if $\# X=2$. Otherwise, let us consider the case that $\# X>2$. To show that $\eta(m)=x$ under the supposition that $\# X>2$, assume, to the contrary, that $\eta(m) \neq x$. Then, $\eta(m)=x \oplus x^{i}$ and $x^{i} \in L\left(\unlhd_{i}^{i-1}, x\right) \backslash\{x\}$, otherwise an immediate contradiction is obtained. Suppose that for some $y \in X \backslash\left\{x, x^{i}\right\}$ it holds that $y \succ_{j}^{*} \eta(m)$ for some $j \in N \backslash\{i\}$. Then, agent $j$ can induce the modulo game by changing $m_{j}$ into $m_{j}^{\circ}=\left(\unlhd_{j}^{j}, \unlhd_{j+1}^{j}, y, k^{\circ j}\right)$. To attain $y$ agent $j$ has only to adjust $k^{\circ j}$ by which he becomes the winner of the modulo game, which contradicts that $m \in \mathbf{N}\left(M, \eta, R\left(\succcurlyeq^{*}\right)\right)$. Otherwise, suppose that, for each $j \in N \backslash\{i\}, \eta(m) \succcurlyeq_{j}^{*} y$ for all $y \in X \backslash\left\{x, x^{i}\right\}$. Suppose that $x^{i} \succ_{j} x$ for some $j \in N \backslash\{i\}$. As $\succcurlyeq_{j}^{*}$ is a 
monotonic extension of $\succcurlyeq_{j}$ it follows that $x^{i} \succ_{j}^{*} x^{i} \oplus x$. By our supposition that $\# X>2$, agent $j$ can induce the modulo game by choosing any $y \in X \backslash\left\{x, x^{i}\right\}$ and changing his message announcement $m_{j}$ into $m_{j}^{\circ}=\left(\unlhd_{j}^{j}, \unlhd_{j+1}^{j}, y, k^{\circ j}\right)$. To attain $x^{i}$ agent $j$ has only to adjust $k^{\circ j}$ by which agent $i$ becomes the winner of the modulo game, contradicting that $m \in \mathbf{N}\left(M, \eta, R\left(\succcurlyeq^{*}\right)\right)$. Then, it holds that $x \succcurlyeq_{j} x^{i}$ for all $j \in N \backslash\{i\}$. Suppose that $x \succ_{j} x^{i}$ for some $j \in N \backslash\{i\}$. Then, agent $j$ can induce the modulo game by choosing any $y \in X \backslash\left\{x, x^{i}\right\}$ and changing his message announcement $m_{j}$ into $m_{j}^{\circ}=\left(\unlhd_{j}^{j}, \unlhd_{j+1}^{j}, y, k^{\circ j}\right)$. To attain $x$ agent $j$ has only to adjust $k^{\circ j}$ by which agent $\ell \in N \backslash\{i, j\}$ becomes the winner of the modulo game, contradicting that $m \in \mathbf{N}\left(M, \eta, R\left(\succcurlyeq^{*}\right)\right)$. Therefore, as $\succcurlyeq_{\ell}$ is a complete preorder for each participant $\ell \in N$, it follows that $x \sim_{j} x^{i}$ for all $j \in N \backslash\{i\}$. It follows that $x, x^{i} \in \bigcap_{j \in N \backslash\{i\}} \max _{\succcurlyeq_{j}} X$ which contradicts the top-coincidence condition. This concludes step 1 , that is, $\eta(m)=x$.

Step 2. $x \in \max _{\succcurlyeq} X$ for all $j \in N \backslash\{i\}$

Suppose that $\# X>2$. Since $m \in \mathbf{N}\left(M, \eta, R\left(\succcurlyeq^{*}\right)\right)$ it follows that $x \in \max _{\succcurlyeq_{j}} X$ for all $j \in N \backslash\{i\}$ as any agent $j$ can trigger the modulo game and obtain any other outcome in $X$. Otherwise, let $\# X=2$. We proceed according to whether $n>3$ or $n=3$. In each of the following sub-cases we show that $x, x^{i} \in \eta\left(M_{j}, m_{-j}\right)$ for each $j \in N \backslash\{i\}$. Since $\eta(m)=x$, by Step 1 , it suffices to show that each agent $j \neq i$ can attain the outcome $x^{i}$.

Sub-case 3.1. \#X $=2$ and $n>3$

Then, agent $j \neq i$ can induce the modulo game by changing his outcome announcement into $x^{i}$ so as to make $\#\left\{\ell \in N \mid x^{\ell}=x^{i}\right\} \geq 2$ and $\#\left\{\ell \in N \mid x^{\ell}=x\right\} \geq 2$. To attain $x^{i}$ agent $j$ has only to adjust the integer index $k^{\circ j}$ by which agent $i$ becomes the winner of the modulo game.

Sub-case 3.2. $\# X=2$ and $n=3$

Then, agent $j \neq i$ can change $m_{j}$ into $m_{j}^{\circ}=\left(\unlhd_{j}^{j}, \unlhd_{j+1}^{j}, x^{i}, k^{\circ j}\right)=\left(\unlhd_{j}, \unlhd_{j+1}, x^{i}\right.$, $k^{\circ j}$ ). We proceed according to whether $j+1=i$ or not.

Sub-sub-case 3.2.1. $j+1=i$

Suppose that $\unlhd_{j+1}^{j} \neq \unlhd_{i}^{i}$. Then, the modulo game is triggered. To attain the outcome $x^{i}$ agent $j$ has only to adjust $k^{\circ j}$ so that he becomes the winner of the modulo game. Otherwise, let $\unlhd_{j+1}^{j}=\unlhd_{i}^{i}$. Suppose that $x^{i} \notin F\left(\unlhd_{j}^{j}, \unlhd_{i}^{i}, \unlhd_{i+1}^{i}\right)$. Thus, the message profile $\left(m_{-j}, m_{j}^{\circ}\right)$ falls into Rule 4 . To attain the outcome $x^{i}$ agent $j$ has only to adjust $k^{\circ j}$ so that he becomes the winner of the modulo game. Otherwise, let $x^{i} \in$ 
$F\left(\unlhd_{j}^{j}, \unlhd_{i}^{i}, \unlhd_{i+1}^{i}\right)$. Suppose that $\left(m_{-j}, m_{j}^{\circ}\right)$ falls into Rule 3 . Then, $\eta\left(m_{-j}, m_{j}^{\circ}\right)=$ $x^{i}$, as sought. Otherwise, $\left(m_{-j}, m_{j}^{\circ}\right)$ falls into Rule 4. Again, to attain $x^{i}$ agent $j$ has only to adjust $k^{\circ j}$ so that he becomes the winner of the modulo game.

Sub-sub-case 3.2.2. $j+1 \neq i$

Then, $j-1=i$. Suppose that $\unlhd_{i+1}^{i} \neq \unlhd_{j}^{j}$. Thus, the message profile $\left(m_{-j}, m_{j}^{\circ}\right)$ falls into Rule 4. To attain $x^{i}$ agent $j$ has only to adjust $k^{\circ j}$ so that he becomes the winner of the modulo game. Otherwise, let $\unlhd_{i+1}^{i}=\unlhd_{j}^{j}$. Suppose that $x^{i} \notin F\left(\unlhd_{i}^{i}, \unlhd_{j}^{j}, \unlhd_{j+1}^{j}\right)$. Again, the message profile $\left(m_{-j}, m_{j}^{\circ}\right)$ falls into Rule 4 . To attain the outcome $x^{i}$ agent $j$ has only to adjust $k^{\circ j}$ so that he becomes the winner of the modulo game. Otherwise, let $x^{i} \in F\left(\unlhd_{i}^{i}, \unlhd_{j}^{j}, \unlhd_{j+1}^{j}\right)$. Suppose that $L\left(\unlhd_{j+1}^{j}, x^{i}\right) \neq X$. Then, $\left(m_{-j}, m_{j}^{\circ}\right)$ falls into Rule 3, and so $\eta\left(m_{-j}, m_{j}^{\circ}\right)=x^{i}$, as sought. Otherwise, $\left(m_{-j}, m_{j}^{\circ}\right)$ falls into Rule 4. To attain $x^{i}$ agent $j$ has only to adjust $k^{\circ j}$ so that he becomes the winner of the modulo game.

Since the above arguments hold for any $j \in N \backslash\{i\}$, we have that $x, x^{i} \in \eta\left(M_{j}, m_{-j}\right)$ for each $j \in N \backslash\{i\}$. Since $m \in \mathbf{N}\left(M, \eta, R\left(\succcurlyeq^{*}\right)\right)$ it follows that $x \in \max _{\succcurlyeq_{j}} X$ for all $j \in N \backslash\{i\}$, otherwise we fall into a contradiction.

Step 3. $x \in F(\succcurlyeq)$

By steps 1 and 2, we have that $\eta(m)=x$ and $x \in \max _{\succcurlyeq_{j}} X$ for all $j \in N \backslash\{i\}$. The top-coincidence condition implies that $\{x\}=\bigcap_{j \in N \backslash\{i\}} \max _{\succcurlyeq_{j}} X$. Then, it is also an equilibrium for all agents to send the message $m^{*}$, where $m_{j}^{*}=m_{j}$ for all $j \in N \backslash\{i\}$ and $m_{i}^{*}=\left(\unlhd_{i}^{i}, \unlhd_{i+1}^{i}, x^{i}, \diamond\right)=\left(\unlhd_{i}^{i-1}, \unlhd_{i+1}^{i+1}, x, \diamond\right)$, so that $m^{*}$ falls into Rule 1 . The same reasoning used above for $m \in \mathbf{N}\left(M, \eta, R\left(\succcurlyeq^{*}\right)\right)$ falling into Rule 1 carries over into $m^{*}$ so that we obtain $x \in F(\succcurlyeq)$, as sought.

Suppose that $m$ falls into Rule 4. Then, $\eta(m)=x^{\ell(m)}$ where agent $\ell(m)$ is the winner of the modulo game. Every agent $i$ can alter the current choice to any other outcome in $X$ by unilateral deviation. Since $m$ is a Nash equilibrium message profile, it follows that $\eta(m) \in \max _{\succcurlyeq_{i}} X$ for all $i \in N$. Top-coincidence implies that $\{\eta(m)\}=\bigcap_{i \in N} \max _{\succcurlyeq_{i}} X$. Weak unanimity implies $\eta(m) \in F(\succcurlyeq)$, as sought.

\section{Concluding remarks}

In this paper, we deal with the informational efficiency issue pertaining to Benoit-Ok's Theorem (Benoît and Ok 2008). We focus on mechanisms in which each agent reports to the planner her own preference and her neighbor's preference solely, in addition 
to a feasible social outcome and an agent index (Saijo 1988). We show that the class of weakly unanimous SCC s that are Nash-implementable by simple stochastic mechanisms endowed with Saijo's message space reduction is fully identified by Maskin monotonicity when the condition of top-coincidence is imposed on environments.

With regard to Nash implementation theory, the main implication of the reported analysis is the following. The class of weakly unanimous SCC s that are Nash-implementable via simple stochastic mechanisms is equivalent to the class of weakly unanimous SCC s that are Nash-implementable by a simple stochastic mechanism endowed with Saijo's strategy space reduction. Note that this result is in line with other well known results of Nash implementation. In particular, the reported equivalence result is analogous to the equivalence relationship between Nash implementation by $s$-mechanisms and Nash implementation in general social choice environments (Lombardi and Yoshihara 2010).

Before closing the paper, we should make one last comment about the result presented here. Our result is built on the implicit assumption that agents involved into a mechanism are selfish and perfectly rational. Dissatisfaction with this classical assumption is mounting. Attempts to replace it with alternative decision models as engines of inquiry into basic economic questions are flourishing. In the light of this recent trend, the reported equivalence relationship may not necessarily hold when a small departure from the "perfect rational man" paradigm is considered.

With regard to Nash implementation, a notion of partial honesty has been introduced and Nash implementation problems with partially honest agents have been studied. A partially honest agent is an agent who has preferences over message profiles and displays concerns for two dimensions in lexicographic order: (1) her outcome and (2) her truth-telling behavior. In the presence of partially honest agents, the equivalence relationship between Nash implementation and Nash implementation by $s$-mechanisms no longer holds, as Lombardi and Yoshihara (2011) show. This suggests that the equivalence relationship indispensably relies on the assumption that agents act purely to advance their own self-interest and are not inclined to attach (moral) rights and duties to their acts. We conjecture that this break-down extends to Nash implementation via simple stochastic mechanisms.

Acknowledgments We are grateful to Hans Peters and Naoki Yoshihara for useful comments and suggestions. Special thanks go to an anonymous referee and an Editor-in-Chief of this journal, whose comments and suggestions have led to substantial improvements in the paper. The usual caveat applies.

Open Access This article is distributed under the terms of the Creative Commons Attribution License which permits any use, distribution, and reproduction in any medium, provided the original author(s) and the source are credited.

\section{References}

Benoît JP, Ok EA (2008) Nash implementation without no-veto power. Games Econ Behav 64:51-67 Danilov V (1992) Implementation via Nash equilibria. Econometrica 60:43-56

Dutta B, Sen A (1991) A necessary and sufficient condition for two-person Nash implementation. Rev Econ Stud 58:121-128

Lombardi M, Yoshihara N (2010) A full characterization of Nash implementation with strategy space reduction (mimeo) 
Lombardi M, Yoshihara N (2011) Partially-honest Nash implementation: Characterization results (mimeo) Maskin E (1999) Nash equilibrium and welfare optimality. Rev Econ Stud 66:23-38

Moore J, Repullo R (1990) Nash implementation: a full characterization. Econometrica 58:1083-1099

Saijo T (1988) Strategy space reduction in Maskin's theorem: sufficient conditions for Nash implementation. Econometrica 56:693-700

Sjöström T (1991) On the necessary and sufficient conditions for Nash implementation. Soc Choice Welfare $8: 333-340$

Yamato T (1992) On Nash implementation of social choice correspondences. Games Econ Behav 4: 484-492 